\title{
Jonsson Comprehensive Cancer Center
}

National Cancer Institute

\section{Source}

National Cancer Institute. Jonsson Comprehensive Cancer Center. NCI Thesaurus. Code C39438.

The Jonsson Comprehensive Cancer Center conducts basic, clinical, and cancer prevention research, while providing the latest treatments to patients, while training the next generation of medical researchers. The Center is developing new technologies for early detection and classification of cancers, combining nanotechnology, systems biology, and molecular imaging. It is affiliated with University of California, Los Angeles and was designated as an $\mathrm{NCl}$ comprehensive cancer center in 1974. 